\title{
PARA QUE NO HAGA EL OSO: HACIA UNA REFLEXIÓN DE LAS PRÁCTICAS DOCENTES A TRAVÉS DEL CINE
}

\author{
Allan Amador Díaz Rueda \\ Especialista en Semiótica y Pedagogía de la Lengua Materna \\ Docente Unidades Tecnológicas de Santander \\ allan.diaz17@yahoo.com
}

\begin{abstract}
Resumen
Desde hace algún tiempo las cintas de dibujos animados han alcanzado un lugar privilegiado en el séptimo arte gracias a magnícas producciones que condensan efectivamente diversos ingredientes que hacen de cada Ime una experiencia agradable no sólo para los niños, sino para toda la familia. El mérito de las nuevas productoras del cine animado radica en una planicación del contenido que permite a estas expresiones cinematográcas ser mucho más que un producto diseñado para divertir; esa es la razón por la que en nuestros días éstas se concentran en presentar historias con una estructura supercial atractiva para los infantes que, simultáneamente, proponen a otros públicos una mayor complejidad de sus tramas con el $\mathrm{n}$ de invitar al espectador a encontrar, luego de un análisis profundo del material audiovisual, sugestivas revelaciones sobre una amplia gama de situaciones sociales, económicas, políticas, culturales e incluso académicas. El presente trabajo analiza cómo esta nueva tendencia de la animación en la pantalla grande tiene cabida en el campo de la educación, pues a lo largo de este documento se mostrará de qué manera la película Kung Fu Panda de los directores Stevenson y Osborne (2001) plantea fuertes cuestionamientos de las prácticas tradicionales en la escuela, a la vez que ilumina, entre líneas, algunas sugerencias que el maestro debería considerar en la práctica diaria de su ocio.
\end{abstract}

Palabras clave: autoanálisis, educar, aprendizaje signicativo, aprendizaje por proyectos, aprendizaje autónomo.

\section{Summary}

Since some time ago, animated movies have reached a special place in cinema thanks to superb productions which eectively condense several ingredients that make each Im an enjoyable experience not only for children, but also for the whole family. The merit of the animated Ims new producers lies in a planning content that allows these Imic expressions to be more than a product designed to entertain; that's the reason why nowadays they are focused on presenting stories with a surface structure attractive to infants that, simultaneously, propose to other audiences greater plots complexity in order to invite the viewer to nd, after a deep audiovisual material analysis, suggestive insights about a wide range of social, economic , political, cultural and even academic matters. This study analyses how this new trend of animation on the big screen has a place in the eld of education due to this document will point out how Kung Fu Panda movie by directors Stevenson and Osborne (2001) poses a number of strong criticisms of traditional practices in the school, meanwhile, between the lines, some suggestions are made about what teachers should take into account in their daily practice of this occupation.

Key words: self-analysis, to educate, meaningful learning, learning through projects, autonomous learning.

"Para enseñar a los demás, primero has de hacer tú algo muy duro: has de enderezarte a ti mismo"

Buda

\section{Introducción}

Cada día los docentes buscan la manera de hacer mejor el trabajo, de hecho siempre se está en busca de claves que nos permitan optimizar el proceso de construcción de conocimiento se esta alerta a las diversas propuestas que complementen y enriquezcan la sin importar de dónde vencharlas y conferencias, se leen artículos y propuestas pedagógicas, se prácticas exitosas de nuestros colegas, se analiza la realidad del país e intenta insertarla en las sesiones, se ensayan nuevas alternativas para hacer de las clases experiencias amenas, creativas, didácticas, interesantes y signicativas. Por ello, de vez en cuando encuentran materiales que a simple vista no ofrecen mayor valor que el de pasar un buen rato en familia pero que, en detalle, proporcionan una serie de elementos que llevan a cualquier maestro a cuestionarse so- 
bre lo que realiza con sus estudiantes. Ese es el caso de la película Kung Fu Panda de los directores John Stevenson y Mark Osborne, publicada en 2008.

DreamWorks y Paramount Pictures produjeron esta cinta que cuenta la historia de Po, un oso panda, hijo de un ganso que se enorgullece de preparar las mejores sopas de deos del Valle de la Paz. Po sueña con ser una leyenda del Kung Fu a la que la multitud adore y honre. No obstante, en cuanto despierta vuelve a la opaca, sin sentido y poco atractiva realidad que vive día a día en el restaurante familiar, la cual soporta sin reparos con el único $\mathrm{n}$ de no defraudar a su padre. Esto cambia cuando se entera que los maestros Oogway y Shi$\mathrm{fu}$, los exponentes más grandes de las artes marciales, elegirán al Guerrero Dragón para defender su pueblo del ataque de Tai Lung, el convicto más temido de la cárcel quien está obsesionado por conocer el secreto que encierra el rollo del Dragón, revelación que dará a quien lo merezca la clave para ser invencible. Es en ese momento cuando el panda decide ir al Palacio de Jade para disfrutar de la competencia entre los cinco furiosos (Tigresa, Mantis, Mono, Grulla y Víbora) que convertirá al ganador en el legendario Guerrero Dragón, el único que podrá vencer a Tai Lung. Por un enrevesado accidente, Po, que había llegado tarde y no podía entrar al evento, se planta justo delante de los maestros. Pero como dice Oogway, los accidentes no existen y Po es elegido el nuevo guerrero dragón. A partir de ese instante, el Ime nos muestra el camino que recorre el panda para cumplir con su destino mientras resalta interesantísimas cuestiones sobre el proceso de formación del individuo.

El primer elemento a considerar es que el maestro Shifu no reconoce en Po un digno aspirante a guerrero dragón. Aquí se muestran los prejuicios e imaginarios que llevan al discípulo a dudar de sus capacidades. ¿Cómo un panda puede llegar a ser el más poderoso de los peleadores? Así se muestra en el siguiente diálogo:

Shifu: -Con que eres el legendario Guerrero Dragón.
Po: -Eso creo.

Shifu: -No. Tú no eres el guerrero dragón y jamás lo serás hasta que aprendas el secreto del rollo del Dragón.

Po: - ¿Cómo funciona? ¿Tiene una escalera o un trampolín?

Shifu: - ¿Crees que es tan fácil? ¿Qué te regalaré el secreto para el poder ilimitado?

-Primero debes dominar el poder más alto del Kung Fu.

-y eso es imposible si es alguien como tú.

Po: - ¿Como yo?

Shifu: -Sí, mírate. Trasero gordo, brazos gelatinosos.

Po: -Eso es sensible.

Shifu: - Y esa ridícula barriga. Además, completo descuido de la higiene personal.

Po: -Un momento, eso es injusticado.

Shifu: -No te me acerques.

Quizá esta misma actitud con diferentes palabras ha estado presente en algunos momentos de nuestra práctica docente, en muchos casos de manera inconsciente. No damos crédito a lo que pueden hacer los educandos cuando asumimos que por su condición socioeconómica, los prejuicios que otros docentes han creado de ellos, su comportamiento conictivo, su desinterés, sus preferencias sexuales o culturales no estarán en capacidad de aprender ecazmente. Esto desemboca en la generación de una imagen negativa que los estudiantes terminan por reconocer como cierta, cuestión que en últimas inuye en su rendimiento académico. En un estudio realizado por (Haslam, Salvatore, Kessler, Stephen \& Reicher 2009), los autores evidenciaron que los imaginarios existentes sobre ciertos grupos sociales pueden interferir en el desempe- 
ño de los individuos que pertenecen a ellos, independientemente de las aptitudes o capacidades que posean. En otras palabras, si una mujer ha escuchado que sus amigos hombres creen que las damas son pésimas conductoras, esa creencia puede afectar su rendimiento durante el examen de manejo y, por consiguiente, impedirá que tenga un desempeño óptimo, aunque disponga de la habilidad y la capacidad para hacerlo.

Lo verdaderamente alarmante de este tipo de comportamientos presentes en nuestra realidad educativa es que se llega al punto de humillar a los estudiantes a través del conocimiento. Así pues, en ocasiones los profesores buscamos conrmar esos imaginarios erróneos sobre nuestros estudiantes enfrentándonos con ellos para mostrarles que somos quienes tenemos el conocimiento de nuestra disciplina y que ellos aún no tienen las competencias necesarias. Una muestra de lo anterior se hace evidente en la escena en la cual Shifu, cansado de la perseverancia del panda ante sus desplantes y esfuerzos por desanimarlo, decide luchar con él para mostrarle precisamente que por más que entrene no tiene sentido insistir en esa tarea, pues nunca podrá convertirse en El guerrero Dragón. Mientras lo golpea con una serie de movimientos complejos propios del Kung Fu le dice:

Shifu: -He procurado que te sea sencillo panda, pero se acabó. Tu siguiente oponente seré yo.

Po: -Genial, eso es.

Shifu: -Un paso al frente. El camino de la victoria es hallar la debilidad de tu oponente y hacerlo que sufra por ella.

Po: -Sí.

Shifu: - Aprovechar su fuerza y usarla en su contra. Hasta que haya caído o se rinda.

Po: -Pero un guerrero de verdad no se rinde, descuide maestro yo nunca me rendiré.

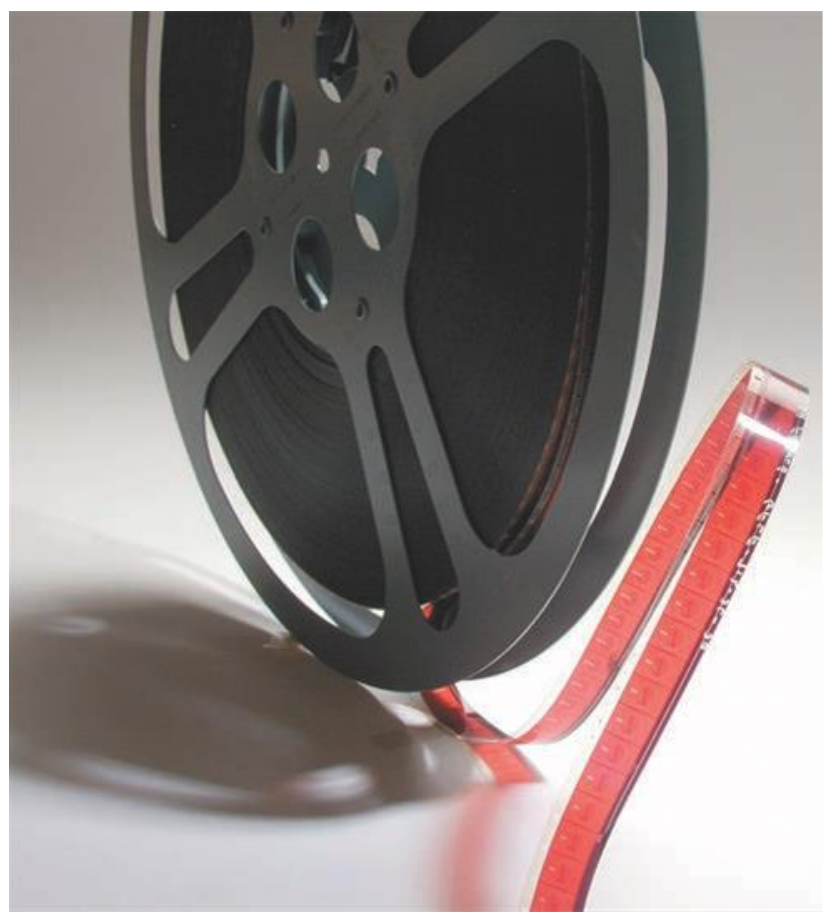

Esta condición ha estado alimentada por la escuela tradicional que promulgaba la dinámica educativa desde la visión del profesor que todo lo conoce y que comparte su sabiduría con sus alumnos, individuos vacíos que asisten al alma mater para ser llenados por sus docentes. Sin embargo, antes de juzgar las actitudes de este tipo de profesores es pertinente revisar algunas prácticas cotidianas. El anterior episodio es fácil de encontrar en las universidades. Aunque no es un fenómeno exclusivo de las instituciones de educación superior, sí es más frecuente hallar docentes que utilizan su amplia trayectoria académica para ejercer una relación de poder en la que el estudiante, a pesar de su entusiasmo por aprender, termina con un profundo sentimiento de frustración e impotencia que lo lleva al fracaso académico.

Razón tenía Freinet (1996) al armar:

No podéis preparar a vuestros alumnos para que construyan mañana el mundo de sus sueños, si vosotros ya no creéis en esos sueños; no podéis prepararlos para la vida, si no creéis en ella; no podríais mostrar el camino, si os habéis sentado, cansados y desalentados en la encrucijada de los caminos. 
Eso es precisamente lo que sucede con algunos profesores. Después de algún tiempo han perdido el horizonte que observa un verdadero maestro en su educandos, piensan que su trabajo no tiene sentido y lo toman como su medio de subsistencia, no como oportunidad para cambiar vidas.

En Kung Fu Panda es posible saber cuáles fueron las causas que llevaron a Shifu a congurar su práctica pedagógica desde la instrucción y no desde la formación. El maestro tuvo una experiencia que marcó su vida. Se entregó en cuerpo y alma a su discípulo favorito: Tai Lung. Este leopardo de las nieves que fue abandonado en la puerta del templo cuando era bebé reunía todas las características que Shifu consideraba necesarias para ser el Legendario guerrero Dragón y así lo entrenó para alcanzar tal honor. No obstante, esto no fue suciente para lograr la meta pues a pesar de haberle enseñado las técnicas más avanzadas del kung fu, su amor de padre putativo no le permitió reconocer las fallas que Tai Lung presentaba en su comportamiento como persona. Como consecuencia de lo anterior, al conocer que no sería nombrado como el Guerrero Dragón Tai Lung destroza el pueblo sin que su maestro pudiera hacer algo para detenerlo. A causa de lo anterior, Shifu decide limitarse a instruir a sus aprendices, cambia su temperamento y siempre mantiene un nivel de exigencia inalcanzable. En conclusión, después de su decepción con Tai Lung, Shifu perdió sus esperanzas de ser exitoso en la búsqueda del verdadero Guerrero del Dragón, por eso dejó de ser un maestro para convertirse en un instructor.

En contraste con Shifu, el maestro Oogway representa la auténtica imagen del maestro que forma a sus educandos. Una ilustración de ello es la conversación que entablan la tortuga y el panda cerca del árbol de durazno:

Oogway: -Veo que encontraste el durazno de la sabiduría celestial.
Po: - ¿Eso era? Perdón, pensé que era un durazno cualquiera.

Oogway:- Te entiendo, sueles comer cuando estás preocupado.

Po: -No estoy preocupado - ¿Por qué habría de estarlo?

\section{Oogway: - ¿Por qué estás preocupado?}

Po: -Di más asco que cualquiera en toda la historia del kung fu. En la historia de China y en la historia del asco.

Oogway: -Probablemente.

Po: - Y los 5... -debió verlos, me detestan con ganas.

Oogway: - ¿Con ganas?

Po: - ¿Qué hará Shifu para convertirme en el guerrero dragón? No soy como los cinco, no tengo alas, ni garras, ni veneno. Hasta Mantis tiene esas cosas. Mejor debería volver a cocinar deos.

Oogway: -Rendirte, no rendirte. Fideos, no deos. Estás preocupado por lo que fue y lo que va a ser. Hay un dicho: "el ayer es historia, el mañana es un misterio, pero el hoy es un obsequio. Por eso se llama presente.

En estas palabras Oogway esboza algunas de las características de un verdadero maestro. Le propongo un breve ejercicio apreciado lector. Con base en algunas ideas tomadas de este parlamento, plantearé algunas preguntas que nos ayudarán a conrmar si efectivamente somos maestros de la talla del creador del Kung Fu.

La primera cuestión para observar tiene que ver con la mística que todo educador debe tener con su profesión. Mientras que Po cree estar comiendo un durazno cualquiera, la tortuga le señala que encontró (es decir, luego de un proceso de búsqueda alcanzó una meta) el durazno de la sabiduría celestial. ¿Alguna vez hemos hecho de nuestra clase una expe- 
riencia extraordinaria? ¿Han sentido nuestros educandos que en cada sesión alcanzan algo signicativo para su vida? ¿Cuántas veces reconocemos los logros, en todos los sentidos, de nuestros escolares?

En segunda instancia, Oogway sintetiza en una frase dos habilidades infaltables de un maestro: la inteligencia para conocer a sus estudiantes y el sentido común para entender las situaciones particulares que influyen su proceso de aprendizaje. "Te entiendo, sueles comer cuando estás preocupado". Oogway se ha tomado el tiempo de observar al panda, lo ha detallado, le ha seguido los pasos; en lugar de compartir el prejuicio que lo marca como incapaz de ser el guerrero dragón, él lo analiza para llegar a entenderlo. Y lo hace (más adelante veremos la relevancia de la comida en el desarrollo de la historia), se toma el tiempo para hablar con Po, valora sus habilidades, conoce sus limitaciones pero sobretodo cree en él. Es oportuno interrogarse ¿Tomamos tiempo para observar a nuestros escolares? ¿Los escuchamos? ¿Reconocemos sus fortalezas y limitaciones? ¿Cada cuánto les mostramos que creemos en ellos?

Además, Oogway no se limita a observar, él indaga, cuestiona, busca respuestas que lo orienten en la comprensión total de su estudiante: ¿Por qué estás preocupado?- pregunta. El mérito de este cuestionamiento radica en un verdadero interés del maestro por entender al estudiante incondicionalmente; tal vez se ha hecho esta misma pregunta pero se queda atónitos al escuchar las respuestas de los escolares. Quiérase o no, muchos de ellos atraviesan por situaciones que en estos tiempos habrían sido impensadas. Aquí radica la capacidad del docente para ser una persona de mente abierta.

Se cree, con Cela \& Palou (2004) que "La escuela ha de ser aquel tiempo y aquel espacio compartidos -sin discriminaciones ni exclusiones de ningún tipo- donde se puedan en- sayar formas de convivencia socialmente útiles y necesarias" No es suficiente con preguntar qué les pasa, si no se está preparado para sus respuestas. Un ejemplo de eso es que muchos profesores se sienten incómodos cuando luego de preguntarle a su estudiante " ¿Por qué estás preocupado?" éste reconoce su homosexualidad en público. En algunas ocasiones esta incomodidad es producida porque el docente tiene la idea que los miembros de la comunidad LGTBI no se ajustan a sus lineamientos culturales, sociales o religiosos. Desde esta perspectiva vale la pena preguntarse: ¿Tenemos todas las herramientas necesarias para comprender sin reparos las situaciones particulares involucradas en los procesos de formación de los estudiantes?

Para terminar el análisis de este diálogo, Oogway no permite que Po renuncie a su sueño; a propósito: ¿Se conocen los sueños, expectativas e intereses de los muchachos? Si es así: ¿cada cuánto los animamos a alcanzarlos? ¿Los usamos para dinamizar la labor docente? ¿Se es en realidad Educador Motivador? ¿Cómo se llega a ser este tipo de maestro?

Puede hablarse que el maestro puede convertirse en un educador motivador (Hernández, 2009) que permite a sus discípulos, tomar decisiones razonables sobre cómo desarrollar las actividades de clase- las cuales están muy ligadas a situaciones reales- y notar las consecuencias de dichas acciones. Por ello, este tipo de orientador atribuye un papel activo al educando en su realización académica, personal y profesional. También, el Docente Motivador es creativo para involucrar a sus muchachos en procesos investigativos, desarrollos intelectuales, acontecimientos o fenómenos de índole individual y social que afecten sus relaciones con la realidad; para obtener lo anterior, el docente reta a los escolares a examinar los contextos que los rodean, evaluar las reglas o normas a las que están expuestos y participar en la dinámica de su formación con el aporte de sus intereses y propósitos explícitos. Dicho de otra manera, 
si un maestro atrae al aula todo lo mencionado propiciará ambientes de aprendizaje ideales que sólo pueden originarse desde la mirada de un Docente Motivador en acción.

Oogway intenta cumplir con todos estos requisitos y lo hace maniesto con su manera de orientar al panda en la búsqueda de su meta. No lo hace directamente, es decir, no da respuestas; simplemente le proporciona una oportunidad para que mediante el uso de todas sus capacidades y habilidades sea el protagonista quien reexione sobre su actuar, identique problemas y plantee soluciones. Así el maestro puntualiza: "Estás preocupado por lo que fue y lo que va a ser. Hay un dicho: el ayer es historia, el mañana es un misterio, pero el hoy es un obsequio. Por eso se llama presente". He aquí una de las contribuciones más signicativas que presenta esta película en términos de educación: siempre se ha hablado que el docente debe explicar de forma clara y las veces que sean necesarias lo que quiere enseñarle a sus estudiantes, cuando existen otras salidas. En realidad lo que los maestros deberíamos hacer es retar a los escolares para potenciar sus habilidades mediante experiencias signicativas que los motiven a aprender. En otras palabras, el rol del maestro no se limita a resolver preguntas, éste tiene el compromiso de abrir las puertas y las ventanas de la curiosidad para que los muchachos salgan a encontrar las respuestas. En palabras de Montessori (1994) "Ésta es nuestra obligación hacia el niño: darle un rayo de luz, y seguir nuestro camino".

Así, en esta pieza cinematográca asistimos al contraste de dos maneras distintas de afrontar la formación: la instrucción, entendida como el conjunto de enseñanzas y prácticas que sirven para el adiestramiento de una persona en un hacer especíco (Shifu), y la educación, proceso de socialización y aprendizaje encaminado al desarrollo intelectual, social y ético de un individuo (Oogway). Es así como, en un momento clave de la historia a través de la concepción del personaje Oogway resalta un hecho educativo que determina por qué un docente no puede transcender la instrucción. Hablamos de la ilusión del control.

Oogway: -Mi viejo amigo, el panda nunca cumplirá su destino ni tú con el tuyo hasta que olvides la ilusión del control.

Shifu: - ¿llusión?

Oogway: -Sí, mira este árbol. No puedo hacer que orezca cuando me place. Ni podré hacer que dé frutos antes de temporada.

Shifu: -Pero hay cosas que podemos controlar. Controlo cuando caerá la fruta. Y controlo dónde plantar la semilla. Esa no es una ilusión maestro.

Oogway: -Sí, pero no importa qué hagas, esa semilla se convertirá en un durazno. Podrás desear un manzano o un naranjo pero tendrás un durazno.

Shifu: -jPero un durazno no puede vencer a Tai Lung!

Oogway: - Tal vez pueda, si estás dispuesto a guiarlo, a nutrirlo, a creer en él.

Shifu: - ¿Pero cómo? Necesito su ayuda, maestro.

Oogway: -No, sólo necesitas creer, prométemelo, Shifu. Prométeme que vas a creer.

Shifu: -Lo intentaré.

Este diálogo resume la importancia que tiene la actitud del maestro en la relación educadoreducando durante el proceso de formación de seres humanos y que De Lózar ( 2011) sintetiza en su libro: "El educador mediocre habla. El buen educador explica. El educador superior demuestra. El gran educador inspira." Tristemente, es todavía usual encontrar en muchas comunidades académicas una admiración excesiva por la disciplina que se encuentra en las clases de los profesores formados bajo un régimen estricto. Para algunos de ellos es motivo de orgullo anunciar desde el inicio del semestre que únicamente cinco estudiantes de los treinta matri- 
culados aprobarán su asignatura. Esto gracias a que son los expertos de la materia que dictan, controlan todo lo que sucede en ella y, por supuesto, a quienes asisten a ella. En los colegios, por ejemplo, muchos padres de familia exigen a los profesores de sus hijos que mantengan la disciplina en clase: las ordenadas, silencio absoluto y el docente como imagen de autoridad. Estas ideas hacen parte de la concepción de la escuela como centro penitenciario en donde se enseña bajo la premisa de tener todo bajo control.

Entonces, si el control en las aulas de clase es real: ¿Por qué Oogway arma que es una ilusión? Un docente que enseña desde esta perspectiva sólo espera que sus estudiantes respondan como máquinas a cuestiones relacionadas estrictamente con su asignatura y así se dará por bien servido. El control es un espejismo en la medida en que quien lo aplica cree que hace una buena labor porque sus estudiantes aprendieron perfectamente lo que él les instruyó, sin tomar en cuenta las implicaciones de ese conocimiento fuera del ámbito académico. Por más que un profesor controle su cátedra, jamás podrá controlar lo que el estudiante aprende, ni mucho menos cómo lo usa en su vida diaria.

Aunque esta ilusión de control ha funcionado tradicionalmente para la trasmisión de conocimientos, nunca será garantía de aprendizaje efectivo; es por eso que con el paso de los años los auténticos educadores se han tomado la tarea de explorar maneras novedosas de formar a sus escolares al brindarles orientación y convicción por lo que son y pueden llegar a ser. Al respecto Reboul (1989) arma que: "Educar no es fabricar adultos según un modelo sino liberar en cada hombre lo que le impide ser él mismo, permitirle realizarse según su 'genio' singular". Dicho de otra manera:¿Qué tal si se reemplazara el control por la convicción? ¿Qué pasaría si enfocamos nuestros esfuerzos no en controlar a los muchachos sino en mostrarles las buenas personas, ciudadanos y profesionales que son y pueden llegar a ser? Meirieu (2006) nos dice:
"(...) hay que darle la vuelta a la representación tradicional según la cual una buena clase es, en primer lugar, una buena actuación del profesor que los alumnos escuchan con atención, en las condiciones que sea. Precisamente, para muchos alumnos, escuchar durante una clase no es tan fácil. Es incluso algo que, a veces, se vive como un sometimiento al poder de un profesor que impone sus caprichos. Entonces, nuestra tarea consiste en hacerles entender que la disciplina no es una manía nuestra, ni una exigencia de la Administración para que "la dejen en paz", sino que emana de las exigencias del trabajo en sí: exigencias concretas, localizadas y compartidas. (Meirieu, 2004, p. 87)

En el caso de Kung fu Panda tenemos las respuesta a estas inquietudes.

Luego de la conversación entre Oogway y Shifu sobre el control, este último reexiona sobre su labor como maestro y comienza un proceso de introspección encaminado a corregir sus errores. El primer paso para conseguir tal objetivo se presenta en el siguiente parlamento:

Shifu: -No puedes irte, un guerrero de verdad nunca se rinde.

Po: -Míreme. Por favor ¿Cómo voy a vencer a Tai Lung? Ni siquiera puedo escapar de usted.

Shifu: -Lo vencerás porque tú eres el guerrero dragón.

Po: - Usted no cree eso, nunca lo creyó, desde que llegué se quiso deshacer de mí.

Shifu: -Sí, eso quería, pero ahora te pido que confíes en tu maestro como yo debo conar en el mío.

Po: -No es mi maestro, y no soy el guerrero dragón.

Shifu: - ¿Y por qué no te rendiste? Sabías que quería deshacerme de ti y te quedaste.

Po: -Sí, me quedé. Me quedé porque cada vez que me lanzaba una piedra o me decía que apestaba 
me dolía pero nunca me dolerá más de lo que me duele todos los días ser yo.

Me quedé porque creí que si alguien podría cambiarme, hacerme... no tan yo, era usted, el mejor maestro de kung fu de toda China.

Shifu: -Pero puedo cambiarte, puedo convertirte en el guerrero dragón y lo haré.

Po: -Por favor, si Tai Lung ya viene en camino. $Y$ aunque se tarde cien años en llegar ¿qué hará usted para convertir esto en el guerrero dragón? ¿Qué? ¿Qué?!

Shifu: - No lo sé! No lo sé.

Po: -Eso pensé.

Lo primero que hace Shifu para enderezarse como maestro es recuperar el respeto de su discípulo, pues a causa de sus errores Po ha dejado de creer no sólo en sí mismo sino en el referente a seguir, a pesar de considerarlo como el mejor representante del kung fu de toda China. Para lograr lo anterior, Shifu intenta demostrar a Po que confía en que él es el Guerrero Dragón. Asimismo, se convence que puede llevar a su educando a la realización que él busca y lo invita a buscar sus sueños juntos. Un mérito más del maestro es reconocer que es humano, que puede equivocarse, incluso que no tiene todas las respuestas o que no tiene idea alguna de cómo cumplirá su misión. Es precisamente en este punto de incertidumbre cuando un gran maestro busca reinventar su práctica, renovarse, mejorar.

Luego de este momento de crisis Shifu tiene la claridad para reconocer cuestiones básicas que todo maestro debe considerar en su práctica diaria. Por ejemplo, después de un proceso de análisis de su estudiante peculiar el maestro ha llegado a la conclusión que no puede enseñarle de la misma manera en la que ha enseñado a sus demás discípulos. Esto me lleva a preguntar: ¿Planeamos y ejecutamos las mismas clases para cada uno de los grupos que tenemos a nuestro cargo sin tener en cuenta las diferencias existentes entre uno y otro?

Howard Gardner postuló la Teoría de las Inteligencias Múltiples en el amanecer de los años ochenta con el objetivo de apoyar los nuevos avances de la ciencia a través de una teoría más analítica de las variadas formas que el intelecto posee para exteriorizarse al momento de aprender; toda vez que, simultáneamente, éste desarrolla el potencial de cada persona de acuerdo a sus talentos naturales. En esta dirección, es necesario recordar que Gardner (1987) advierte que el ser humano posee siete tipos de inteligencias: lingüística, lógico-matemática, musical, espacial, kinestesicocorporal, interpersonal e intrapersonal. Esta propuesta se ha mantenido vigente a pesar de la constante asignación que los expertos en aprendizaje realizan cada día sobre nuevas inteligencias como la espiritual propuesta por Gallegos (2005) o la digital desarrollada por Cobeña Fernández(2010), sólo por mencionar algunos casos. Sin embargo, este autor ha mantenido su carácter de pionero gracias a que concibió la inteligencia de los seres humanos como un potencial que no puede ser encasillado en los cánones instituidos por la escuela tradicional en la que primaban la memorización y acumulación descontextualizada de información. En esencia, Gardner fue uno de los primeros en identicar que a estudiantes con habilidades innatas distintas, procesos de estimulación diferentes para optimizar sus aprendizajes.

A la luz de lo anterior, una vez el maestro es consciente del reto que constituye enseñarle a un estudiante distinto a los demás-así deberíamos apreciarlo-, Shifu se toma el tiempo necesario para descubrir la llave que le permita a Po aprender lo necesario para convertirse en el Guerrero Dragón. Este proceso da buen resultado al identicar que ese elemento facilitador del aprendizaje será algo por lo cual el panda hará lo que sea: la comida. Aquí encontramos uno de los principios del aprendizaje signicativo propuesto por Ausubel (1976) y que es 
puesto en práctica de manera maravillosa a lo largo de una de las mejores secuencias de la película: el entrenamiento. En éste, Shifu no sólo hace uso de la ansiedad generada por el hambre para exigir a Po, sino que también comprende la importancia de romper las barreras establecidas por el salón de clase y busca espacios donde se desarrolle acertadamente el proceso de enseñanza -aprendizaje. Cabe enfatizar que la trama deja ver que la preparación para la batalla nal se lleva a cabo en un paisaje armónico, pacíco y pertinente para tal misión, por eso justamente allí fue donde nació el Kung fu. Después de resaltar lo anterior pregunto: ¿Será que insistimos en enseñar las partes de la planta con un ache dentro del salón o preferimos hacerlo a través de una exploración del jardín del colegio? A propósito: ¿Por qué es importante que el estudiante aprenda las partes de la planta?

Acerca de la pertinencia de aquello que se enseña y se aprende, no cabe duda que en Kung Fu Panda se muestre que todo esto depende de la utilidad que tenga en la vida real. Lo aprendido por Po durante el entrenamiento, tiene sentido en la medida en que sea efectivo para derrotar a Tai Lung en la batalla nal que permitirá salvar El valle de la Paz. Ahora bien, una de las cuestiones a destacar de este entrenamiento no es sólo que es en extremo entretenido para el espectador, sino que fomenta el aprendizaje como desafío. Esta parte de la cinta presenta de manera metafórica la sana tensión que debe existir entre el maestro que utiliza un elemento signicativo para sus estudiantes y el educando que debe explotar sus particularidades para lograr sus objetivos de aprendizaje. Sobre este apartado es importante subrayar que un verdadero maestro demanda a los escolares desplegar lo mejor de ellos mismos para alcanzar sus metas. Así, Cela \& Palou (2004, p.19) sostienen que "La labor del maestro es una cuestión de ética. La labor del maestro es una cuestión de arte". En esa medida, se pueden identicar y difundir las habilidades excep- cionales de cada uno de los muchachos para reconocer sus talentos, aportes, logros y proyecciones a futuro. Cabe anotar que esta idea del aprendizaje como desafío contiene un aditamento especial, se trata de la promoción de estrategias para la resolución de problemas no sólo del campo disciplinar de cada asignatura, sino en la vida cotidiana. Esta escena representa lo que deberíamos hacer en cada uno de los encuentros con nuestros educandos.

Otro aporte para nuestra práctica docente que se desprende del entrenamiento entre Shifu y Po es que en éste el maestro Shifu incluye todos los sentidos: visión, olfato, gusto, tacto, oído, y kinestesia. Todo esto muy propio del proverbio chino que sentencia "Dime y olvido, muéstrame y recuerdo, involúcrame y comprendo". Desde esta perspectiva si involucramos a los estudiantes en un entrenamiento en el que se mezclen diversos elementos de lo físico, lo lógico, lo actitudinal, lo social y lo losó$\mathrm{co}$, entre otros aspectos, aseguraremos mejores procesos de enseñanza -aprendizaje. De esta manera se podrá alcanzar verdaderamente la bastante promocionada y poco lograda formación integral la cual, parte del reconocimiento público de los alcances de los muchachos. Lo digo porque en algunas oportunidades los docentes olvidamos lo que signica para un escolar que se ha esforzado escuchar de los labios de su profesor "excelente trabajo, felicitaciones". Sólo si se valoran los logros que cada estudiante alcanza podemos esperar que el educando valore lo que ha aprendido.

Un aprendizaje más para la praxis educativa que podemos tomar de la película es que si tenemos en cuenta todo lo anteriormente mencionado podremos fomentar en nuestros educandos algunos elementos del aprendizaje autónomo mencionados por Bernardo(2011) en la compilación que al respecto realiza en el texto Aprendizaje autónomo: orientaciones para la docencia. En él, se sugiere que el profesor debe siempre generar un ambiente positivo y de respeto al interior del aula para facilitar que 
los escolares puedan resolver retos alcanzables y tareas que estén dentro de su nivel de formación. De allí la importancia que tiene el educador como alguien que confía en sus estudiantes y, por ello, los motiva a conseguir altas expectativas. Para ello es trascendental que el maestro enseñe a los escolares a observar el trabajo que los demás realizan con el propósito de identicar cómo logran sus metas de aprendizaje. Esta estrategia no sólo incentiva el trabajo grupal, sino también retroalimenta grupal e individualmente al curso, con el n que cada estudiante realice procesos de cognición y metacognición que lo lleven a extender su proceso de formación fuera de la escuela. En complemento, se requiere que el maestro estimule por medio de mensajes positivos a quienes presentan autoecacia baja, a la vez que realiza críticas constructivas y sugerencias para mejorar lo que hacen, siempre bajo el reconocimiento de los esfuerzos realizados, nunca con la vergüenza o humillación grupal como herramienta de exigencia.

Es después de insertar estos procesos pedagógicos en el aprendizaje de los sujetos que puede darse la autonomía, pues existen cuestiones que únicamente el estudiante puede descubrir luego de una buena orientación por parte del maestro, la cual le ha facilitado al educando la generación de herramientas claves para reforzar sus aprendizajes fuera del aula de clase. Una prueba contundente de este señalamiento se muestra al nal de la batalla entre Po y Tai Lung en la que el panda aplica a su enemigo una de las armas más temidas del kung fu: la llave dactilar Wuxi.

Tai Lung: -iLa llave Dactilar Wuxi!

Po: - ¡Oh! ¿Conoces esta llave?

Tai Lung: - ¡Estás mintiendo! ¡Estás mintiendo! ¡Shifu no te enseñó eso!

Po: -Nop. Lo deduje solo. jSkadoosh!

Cuando Po ejecuta -luego de aprender au- tónomamente- una de las técnicas superiores de las artes marciales (la llave dactilar Wuxi) llegamos al desenlace de la historia donde él nalmente es reconocido por toda la comunidad, incluso por los cinco furiosos, como el Legendario Guerrero Dragón que ha salvado a su pueblo. Es decir, nalmente el panda ha alcanzado sus sueños y ese logro personal ha impactado de manera positiva a su comunidad. Ése es el tipo de aprendizaje que logra modicaciones en la realidad inmediata de los pueblos, la misma que está contemplada en los Objetivos Básicos de la Educación propuestos por la UNESCO (2005): aprender a aprender, aprender a resolver y aprender a ser. Revisemos si el héroe de Valle de la Paz cumple con estas condiciones. A lo largo de su entrenamiento Po ha reconocido que aprende mejor cuando motiva sus acciones en torno a la comida (metacognición), es capaz de potenciar sus capacidades con el $n$ de derrotar a Tai Lung (resolución de problemas) y reconoce que es un ser excepcional que puede inuir en su entorno (autoreconocimiento y competencias ciudadanas), la prueba reina que demuestra esto último la encontramos durante la batalla denitiva:

Tai Lung: -"No puedo ser derrotado por un panda grande y gordo"

Po: -"No soy un panda grande y gordo, soy $E \underline{L}$ panda grande y gordo".

Finalmente Shifu ha logrado en Po lo que tantas veces hemos escuchado en una variedad de discursos académicos: la formación integral que al decir de Ospina (2012) es la pregunta y consecuente respuesta sobre qué es el saber: "A veces algo que tiene que ver con la memoria, a veces, con la destreza, a veces, con la recursividad" (p.20)

\section{Conclusión}

El camino recorrido por Po constituye un ejemplo de aprendizaje integral en el que se pueden delinear condiciones básicas para de- 
sarrollos educativos ideales; así pues, es posible establecer de acuerdo a estas reexiones que los procesos de aprendizaje deben estar centrados en el estudiante, para ello, se requiere tomar en cuenta sus ritmos para aprender; adicionalmente, se puede apreciar el esfuerzo que los maestros debemos realizar para desarrollar la habilidad de identicar y utilizar en nuestras prácticas contenidos signicativos del mundo real que potencie las capacidades de los aprendices. Además, podemos identicar que nuestras acciones pedagógicas deben estar enmarcadas en contextos reales de interacción en el entorno, razón por la cual es necesario buscar que los aprendizajes de nuestros niños y jóvenes contribuyan a solucionar problemas de sus comunidades. Con el ánimo de lograr lo anterior, tenemos que ser conscientes de la necesidad de establecer objetivos especícos que articulen el aprendizaje de las diversas disciplinas de estudio con la formación como ser social del individuo para que los logros de nuestros muchachos puedan compartirse con la sociedad. Es así como, gracias a experiencias educativas exitosas se establecen y hacen maniestas conexiones entre lo académico, lo social, lo personal y lo profesional que propicien oportunidades para la reexión, el autoconocimiento y la autoevaluación por parte del estudiante y que redunden en la conguración de su aprendizaje autónomo.

El Ime Kung Fu Panda constituye una invitación abierta a los maestros para que realicen constantes procesos de autoevaluación sobre nuestras prácticas docentes que propendan por el mejoramiento de nuestra labor y nos permita ser más efectivos para ayudar a nuestros estudiantes a aprender.

Mención aparte para la escena que los productores dispusieron para despedir al espectador después de los créditos. En ella, maestro y guerrero dragón comparten una comida al lado del durazno mientras la toma se cierra para resaltar el crecimiento de un nuevo árbol y así recordarnos que nuestra labor nunca termi- na, que es cíclica y, por ende, siempre debemos estar prestos a encontrar en los lugares menos esperados herramientas novedosas para conrmar que somos verdaderos maestros.

\section{Bibliografía}

Ausubel, D. P. (1976): Psicología Educativa. Un punto de vista cognoscitivo. Primera edición. Trillas, México.

Ausubel, D. P. \& Novak, et. al. (1983) Psicología educativa. Un punto de vista cognoscitivo. México: Trías

Cela, J y Palou, J. (2004). Carta a los nuevos maestros. Barcelona: Paidós.

Cobeña Fernández, J.A. Inteligencia rápida, inteligencia tranquila. Disponible en: http://www.joseantoniocobena.com/?author $=1$ [Consultado 20 de julio de 2012]

Crispín, Bernardo, M. L. (comp) Aprendizaje autónomo: orientaciones para la docencia. Universidad lberoamericana, AC. Primera edición electrónica: 2011. México D.F. Disponible en: http://www.uia.mx/web/ les/publicaciones/aprendizaje-autonomo.pdf [Consultado, mayo 16 de 2012]

De Lózar Cuevas, E. El docente Ideal. Publicado en enero 10 de 2011. Disponible en: http://laplumacritica. blogspot.com/2011/01/el-docente-ideal.html [Consultado. Junio 18 de 2012]

Dickinson et al., 1998; Katz \& Chard, 1989; Martin \& Baker, 2000; Thomas, 1998. NorthWest Regional Educational Laboratory. Aprendizaje por proyectos. Publicado en EDUTEKA. Marzo 11 de 2006 Disponible en: http:// www.eduteka.org/AprendizajePorProyectos.php [Consultado, junio 18 de 2012]

Gardner, Howard (1987), Estructuras de la mente. La teoría de las múltiples inteligencias, México: Fondo de Cultura Económica.

Freinet, C. (1996), La escuela moderna francesa - Una pedagogía moderna de sentido común - Las invariantes pedagógicas. Madrid. Ediciones Morata.

GALLEGOS NAVA, R. (2005) Educación y Espiritualidad. La educación como práctica espiritual. Ed. Fundación Internacional para la Educación Holista. Guadalajara, México.

Meireu, P. (2006) Carta a un joven profesor. Barcelona: Grao. 
Montessori M. (1994) Formación del hombre. México. Diana.

Ospina, W. (2012). La lámpara maravillosa. Cuatro ensayos sobre la educación y un elogio de la lectura. Mondadori: Colombia.
Organización de las Naciones Unidas para la educación, la ciencia y la cultura.(2005) Objetivos de desarrollo del milenio: una mirada desde América Latina y el Caribe. Disponible en: http://www.unesco.org/es/education-for-sustainable-development/strategy/learning-to-be/ [Consultado el día 26 de julio de 2012]

Cómo citar este artículo:

Díaz, A. A. (2012). Para que no haga el oso: Hacia una reexión de las prácticas docentes a través del cine. Espiral, Revista de Docencia e Investigación. 2, (1), 41 - 52 
